\title{
Cognitive Bias Modification Training in Adolescents: Persistence of Training Effects
}

\author{
Stefano R Belli; Jennifer Y F Lau \\ Psychology Department, Institute of Psychiatry, Psychology \& Neuroscience, London SE5 8AF \\ Department of Experimental Psychology, South Parks Road, Oxford, OX1 3UD
}

\begin{abstract}
Negative biases in the interpretation of social information are associated with anxious symptoms in adolescents. Previous studies have attempted to modify interpretive biases to alleviate anxious mood responses but the longevity of such training effects has not been established. A Cognitive Bias Modification of Interpretations (CBM-I) paradigm was administered to sixty-nine 15-17 yearolds. Participants were either trained to interpret ambiguous social situations positively, or received control training that contained no emotional content. Participants showed significantly weaker endorsement of negative interpretations of novel ambiguous information following positive training than following control. Positive CBM-I training effects on interpretation did not differ between a group tested immediately following training and one tested 24 hours later. Results provided no evidence of differential changes in state anxiety as a direct result of CBM-I training. The persistence of training effects of suppressing negative biases for ambiguous social information is encouraging for future work that might use bias-training methods in adolescents as buffers against negative emotional responses.
\end{abstract}

Keywords: Adolescence; cognitive bias; CBM-I; interpretation; anxiety 
Anxiety problems are common in youth (Costello, Egger, \& Angold, 2005; Rapee, Schniering, \& Hudson, 2009), causing impairment at significant lifetime cost (American Psychiatric Association, 2000; Meltzer, Gatward, Goodman, \& Ford, 2000). Many incidences of adult anxiety show first onset between 11 and 15 years (Gregory et al., 2007; Kim-Cohen et al., 2003; Rohde, Lewinsohn, \& Seeley, 1991), highlighting late childhood and adolescence as a sensitive period for the emergence of persistent anxiety. There have been many neurobiological and psychosocial explanations why the transition to adolescence may be associated with anxiety onset (Haller, Cohen Kadosh, \& Lau, 2013). However, we have also recently argued that on the basis of typical neurodevelopmental and experiential changes in this period - notably the process of experience-dependent pruning and associated plasticity - there may also be increased responsiveness to beneficial interventions in adolescence (Cohen Kadosh, Linden, \& Lau, 2013; Lau, 2013). A next obvious question is what interventions may be most appropriate for targeting anxiety in this age range.

Cognitive Bias Modification of Interpretations (CBM-I) training has emerged as a possible interventive tool. It draws on basic cognitive science findings of links between interpretive biases-the tendency to draw negative interpretations from ambiguous information - and symptoms of anxiety in adults (Mathews \& MacLeod, 2002), but also in children and adolescents (Field \& Lester, 2010; Hadwin \& Field, 2010; Hadwin, Frost, French, \& Richards, 1997; Vassilopoulos \& Banerjee, 2008; Warren, Emde, \& Sroufe, 2000). Beyond such concurrent associations, data suggest that negative interpretations may precede onset of anxiety symptoms, for example in offspring of anxious parents (Creswell, OConnor, \& Brewin, 2006; Creswell, Schniering, \& Rapee, 2005). If interpretive biases can be modified through training interventions, it could be possible to counteract their negative effects. Various training paradigms that modify interpretational styles have therefore been developed (Suway \& Fox, 2012). In most of these, participants are provided with emotionally ambiguous scenarios, and encouraged (with feedback) to resolve these scenarios positively across training trials.

While most CBM-I studies have been conducted in adults across healthy, analogue and clinical samples, various studies have also extended these paradigms to children and adolescents, generally demonstrating the malleability of interpretive biases at these ages. In particular, CBM-I training has been shown to give rise to benign or actively positive post-training interpretations of novel ambiguous information, compared to comparison conditions that are developed to either not change interpretations (test-retest control groups, neutral training groups) or foster negative interpretations (negative CBM-I training). Such findings have been demonstrated in child (Muris, Huijding, Mayer, Remmerswaal, \& Vreden, 2009; Vassilopoulos, Banerjee, \& Prantzalou, 2009) and adolescent samples (Lothmann, Holmes, Chan, \& Lau, 2011; Salemink \& Wiers, 2011), targeting social fears (Lau, Molyneaux, Telman, \& Belli, 2011), animal fears (Lester, Field, \& Muris, 2011a, 2011b) and more general fears (Muris et al., 2009). CBM-I has been also been shown to alter interpretational styles in adolescent patient samples (Fu, Du, Au, \& Lau, 2013).

Effects of training on reducing anxious mood have been less consistent, but nonetheless present in some studies. Such effects have been seen in both adult (Lang, Blackwell, Harmer, Davison, \& Holmes, 2012) and child/adolescent samples (Lau et al., 2011; Lau, Pettit, \& Creswell, 2013; Lothmann et al., 2011; Vassilopoulos et al., 2009; Vassilopoulos, Blackwell, Moberly, \& Karahaliou, 2012). Other work has shown CBM-I training to buffer against anxious responses to stressors (Lau, Belli, \& Chopra, 2013) in typically-developing adolescents.

While many prior studies have shown that adaptive interpretive styles can be generated through training in children and adolescents with some effects of symptom reduction, a major outstanding question is whether these beneficial effects on interpretations persist over time. This is critical if CBM-I is to be considered a potential intervention for young people with anxiety problems, as the postulated mechanism for action of the training process involves individuals taking newly-modified interpretation biases and applying them to their subsequent day-to-day lives (Hoppitt, Mathews, Yiend, \& Mackintosh, 2010; MacLeod \& Holmes, 2012). A preliminary test of the perseverance of positive training effects would be to investigate whether these last for 24 hours, for which there is some evidence in adults. Yiend, Mackintosh and Mathews (2005) showed that 24 hours after positive CBM-I training participants endorsed more positive than negative interpretations of novel ambiguous material, whereas the comparison training condition (participants undergoing negative training) showed the inverse effect. These findings were subsequently replicated by Mackintosh, 
Mathews, Yiend, Ridgeway and Cook (2006) in another sample of healthy volunteers. Indications of similar longevity of training effects has also previously been shown in late childhood (Vassilopoulos et al., 2009, 2012) but not yet in adolescents.

The present study aims to examine whether the effects of a single session of CBM-I training persist over a 24 hour period in an adolescent sample relative to a neutral (control) training condition. These results have previously been demonstrated in adults (Mackintosh et al., 2006; Yiend et al., 2005), but given the large developmental differences between adolescents and adults in social and interpersonal cognition (Blakemore, 2008; Steinberg \& Monahan, 2007) and apprehension and retention of various forms of information (Blakemore \& Choudhury, 2006), it is not certain that the same results would be found during adolescence. Nevertheless, it is hypothesised that relative to neutral control training, CBM-I training will suppress negative endorsements of ambiguous novel information, and may also potentiate endorsement of positive interpretations. Further it is hypothesised that these post-training effects will persist over a 24-hour period following CBM-I training, as shown by Yiend et al. (2005) and Mackintosh et al. (2006) in adults. Using a single training session allows for an examination of the discrete effects of CBM-I training itself upon interpretation, while limiting potential interference from intervening experiences of daily or life events (see: Hoppitt et al., 2010). Mood effects are expected to be congruent with training valence - i.e. positive training will decrease state anxiety, while no changes are expected following neutral control training. Previous research has shown the clearest effects of CBM-I on state mood to be seen on anxiety measures (e.g. Lau et al., 2011; Lothmann et al., 2011; Vassilopoulos et al., 2009), but state measures of low mood and positive affect will also be included in the current study as secondary analyses.

\section{Methods}

\section{Participants}

Sixty-nine participants were recruited from mixed and all-girls state schools in Oxfordshire and London $\left(\bar{x}_{a g e}=15.68, s d=1.53 ; 79.7 \%\right.$ female $)$. Participants were only recruited if they had no experience of mood or anxiety disorders, as individuals diagnosed with anxiety or mood problems may have more complex patterns of co-morbidity (Hettema, Prescott, \& Kendler, 2003; Moffitt et al., 2007; Rohde et al., 1991), and previous/current treatment, which might potentially complicate the interpretation of results. Participants were randomised to one of three conditions for the study: neutral control training $(n=23)$, positive CBM-I training tested immediately following training $(n=24)$, and positive CBM-I training tested 24 hours following training $(n=22)$. Further details of the study design are given below.

\section{CBM-I Training}

The computerised CBM-I training followed Lothmann et al. (2011)'s paradigm. Training consisted of 5 blocks, each containing 12 training items. An example item is given in Figure 1. Each item began with a short paragraph describing a social scenario relevant to adolescents, but missing its final word. When participants finished reading the paragraph, they pressed the spacebar to reveal a word fragment that they were required to complete. Participants had to type the first of the fragment's missing letters to advance to the next screen, thereby ensuring that they had understood the final word of the scenario. The completed word fragment resolved the scenario positively, negatively, or neutrally. Completion of the word fragment was followed by a comprehension question that reinforced the valence of the training item with 'Correct!' or 'Wrong!' displayed on-screen as feedback. For neutral resolutions, comprehension questions were simply factual (see Figure 1). The neutral control scenarios were designed and written to make sure as far as possible that any ambiguities were eliminated from the materials produced, as well as positive or negatively-valenced content. An example positive/negative scenario was:

"One of your teachers asks everyone to come up with ideas for the school play. When you are asked to give your ideas, you think your classmates will find them..." 


\section{[exciting/unoriginal]}

The corresponding neutral scenario did not solely change the final word to e.g. "okay". Rather in this instance, the scenario was slightly re-written:

"One of your teachers asks everyone to come up with ideas for the school play. When you are asked to give your ideas, you say them to the rest of your..."

\section{[classmates]}

Thus, while positive and negative resolutions were aimed to create and reinforce positive and negative interpretations of the ambiguous situations, neutral resolutions did not create valenced interpretations. Accuracy and reaction times of responses to comprehension questions were recorded for each item to allow for investigation of how performance varied over the course of training.

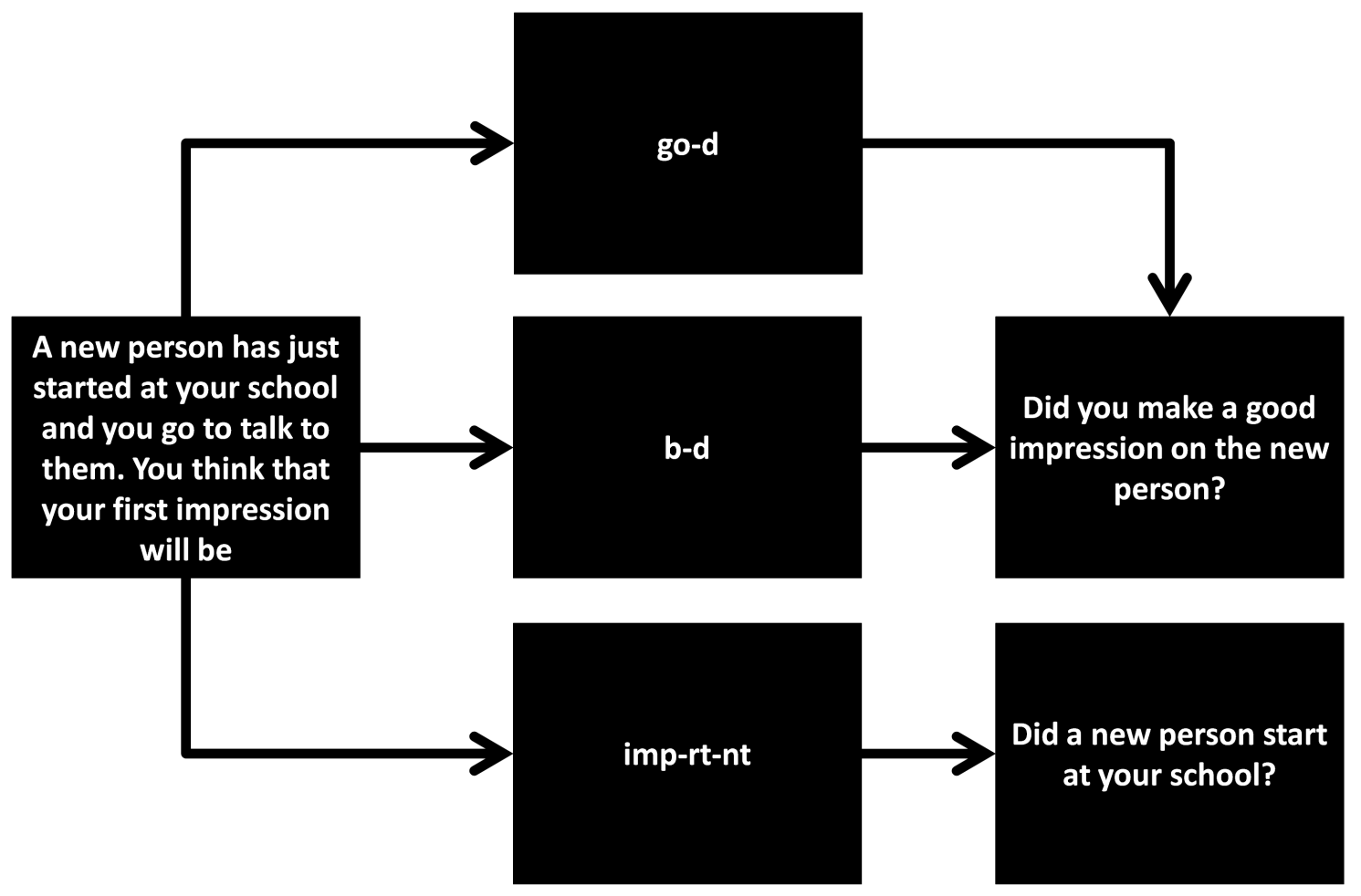

Figure 1: Example of a CBM-I trial, including (from left to right) the ambiguous scenario, word fragment, and comprehension question. Resolutions are, from top to bottom: positive, negative, neutral.

Participants were randomly allocated to one of two training paradigms: positive CBM-I or neutral control. For participants allocated to receive positive CBM-I, 10 items in each training block were resolved positively, one resolved negatively, and one neutrally. This approach was taken in preference to simply having $100 \%$ positive items in an attempt to make the nature of training less readily apparent, thereby mitigating potential demand characteristics. The neutral control featured 10 neutral resolutions, one positive, and one negative per block. 


\section{Measures}

\section{State-Trait Anxiety Inventory for Children (pre-training)}

The trait scale of the State-Trait Anxiety Inventory for Children (STAIC; Spielberger, 1973) was used to ensure there were no differences in anxiety symptoms between experimental groups prior to CBM-I training. The STAIC trait scale comprises 20 self-report items pertaining to anxious symptoms (e.g. 'I worry too much', 'I am secretly afraid') that participants rate for their frequency of experience. Ratings (Hardly Ever, Sometimes, Often - coded 1, 2, and 3 respectively) are summed across items to give trait anxiety scores ranging 20-60. In the current dataset the measure had excellent internal reliability (Cronbach's $\alpha=.86$ ).

\section{Ambiguous Social Scenarios Interpretation Questionnaire (pre-training)}

Eight items deemed suitable for use in adolescents were taken from the Ambiguous Social Scenarios Interpretation Questionnaire (ASSIQ; Amir, Foa, \& Coles, 1998; Stopa \& Clark, 2000). This scale was administered to ensure there were no differences in the endorsement of negative interpretations of ambiguous social scenarios between experimental groups prior to CBM-I training. Each item presented an ambiguous scenario with a choice of three potential explanations, one of which was always a negative reflection on the participant while the other two corresponded to benign interpretations. Participants were instructed to select what they felt was the most likely explanation, e.g. 'You go into a shop and the assistant ignores you. Why do you think this is?' (i) They are bored with their job, and behave rudely (benign); (ii) They are concentrating on something else (benign); (iii) You are not important enough for them to bother with (negative). Responses to each item were coded ' 1 ' if participants endorsed the negative interpretation from the three choices, and ' 0 ' otherwise. This gives a measure of social interpretive bias that ranges $0-8$. The measure's internal reliability in the current study was however lower than would be desirable: $\alpha=.56$.

\section{Interpretation Bias Test (post-training)}

The Interpretation Bias Test was taken from Eysenck et al. (1991), via Mathews and Mackintosh (2000). Participants read 10 novel scenarios structured and presented similarly to training items. However, in contrast to training, scenarios remained ambiguous after completing the final word and comprehension question, and each scenario was additionally given a title, e.g. 'Giving a Speech'. Again the paradigm was adapted from earlier CBM-I work (Lothmann et al., 2011) to make content more relevant to adolescence.

Following the 10 scenarios, the titles of each were presented again with four probe statements, which participants rated for similarity to the original scenario on a scale from 1 (not similar at all) to 4 (very similar). The four probe statements always contained one valid negative interpretation of the original scenario (Negative Target), one valid positive interpretation (Positive Target), one negative statement that was not directly related to the original scenario (Negative Foil), and one positive statement unrelated to the original scenario (Positive Foil). Similarity ratings of these four kinds of statements allowed for analyses of the endorsement of positive versus negative interpretations of novel ambiguous scenarios following training (Targets), compared to measures of tendency to simply endorse non-specific positive and negative statements (Foils). Based on previous studies in adolescents, target and foil probes were be analysed separately to provide insight into the direction of any interaction effects - e.g. interactions between probe valence, probe type (targets/foils) and training valence (Lau et al., 2011; Lothmann et al., 2011).

\section{Visual Analogue Scales for Mood (pre-training, post-training, pre-test)}

State anxiety, positive affect and low mood ratings were assessed over the experiment (see Procedure) using Visual Analogue Scales (VAS). At each presentation of the VAS, participants were given a sheet of paper with 12 items; each featured a $10 \mathrm{~cm}$ line labelled 'I feel not [emotion] at all' and 'I feel very [emotion]' at the left and right extremes respectively. Scores were coded 0.0-10.0, based on where participants crossed the line for each item. Four items pertaining to anxiety (nervous, 
upset, worried, anxious), four items pertaining to positive affect (happy, calm, cheerful, energetic), and four items corresponding to low mood (sad, miserable, upset, gloomy) were drawn from the Positive and Negative Affect Scale for Children (Laurent et al., 1999). Means were calculated across each of the three sets of four items to generate state anxiety, positive affect and low mood ratings at pre-training, post-training, and pre-test for all participants. Of note, the 24-hour group completed one additional set of ratings after the distractor task at the end of their first session; this was simply to ensure that their mood returned to baseline before leaving and was not analysed. Given that participants were exposed to a variety of ambiguous social information over the course of training, we were conscious that there was a possibility that any participants at high risk for social anxiety traits could potentially find the training stressful. Accordingly, scores before and after training were eyeballed for any drastic changes in mood as a result of training - if any severe changes had occurred then participants would have been offered a 'remedial' extra session of positive training following the experiment, as outlined in Lothmann et al. (2011). Reliability was at least fair, and generally very good for the three scales at each time-point: pre-training $\left(\alpha_{\text {Anxiety }}=.86 ; \alpha_{\text {PosAffect }}=.65\right.$; $\left.\alpha_{\text {LowMood }}=.89\right)$, post-training $\left(\alpha_{\text {Anxiety }}=.84 ; \alpha_{\text {PosAffect }}=.82 ; \alpha_{\text {LowMood }}=.87\right)$, and pretest $\left(\alpha_{\text {Anxiety }}=.81 ; \alpha_{\text {PosAffect }}=.80 ; \alpha_{\text {Low Mood }}=.90\right)$-and excellent overall $\left(\alpha_{\text {Anxiety }}=.91 ;\right.$ $\left.\alpha_{\text {PosAffect }}=.92 ; \alpha_{\text {LowMood }}=.94\right)$.

\section{Procedure}

After written parental and participant consent was obtained, participants completed demographic information and pre-training measures of trait anxiety symptoms, interpretational style, and state anxiety. Participants were then randomly allocated to either positive CBM-I training or neutral control. Of those participants who were allocated to receive positive CBM-I training, half completed post-training measures immediately, and half were asked to come back after 24 hours to complete these measures. Accordingly, twice as many participants were allocated to the positive CBM-I training than the neutral control.

Immediately prior to training, participants engaged in a short imagery exercise to enhance the subsequent effects of CBM-I, based on Holmes, Mathews, Dalgleish and Mackintosh (2006) and Holmes, Lang and Shah (2009). This encouraged participants to imagine scenarios happening 'through their own eyes', and was referred back to during the training process via the prompt 'Imagine the events as happening to yourself', displayed after each training block. After CBM-I training, participants completed a second set of post-training VAS measures. A distractor task was then administered to eliminate any potential between-group state mood effects as a result of training. This lasted for 10 minutes, and consisted of rating 60 pictures (abstract shapes, household objects and natural scenes) for pleasantness, each during a 10-second window. Following the distractor task all participants again completed a third set of VAS ratings to ensure that moods had returned to baseline.

After this point, all participants who underwent neutral training completed the Interpretation Bias Test. However, following the third set of VAS ratings, participants who underwent positive CBM-I training diverged into two groups. Half completed the Interpretation Bias Test immediately after the ratings ('Same-Session' CBM-I group), as in the neutral group. The other half left, returning 24 hours later to complete a fourth set of state anxiety ratings followed by the Interpretation Bias Test ('24-Hour' CBM-I group). After completing the Interpretation Bias Test, participants were debriefed in full, thanked and reimbursed for their time. Participants were tested in a university laboratory setting, and the entire course of the experiment took $\sim 1 \mathrm{hr}$. In the case of the 24 -Hour group, the first session took roughly 45 minutes, and the Interpretation Bias Test session on the second day lasted approximately 15 minutes.

Ethical approval for the current study was obtained via the University of Oxford Central University Research Ethics Committee (CUREC). Schools and headteachers were contacted, informed of the nature of the study, and given the opportunity to ask any questions of the researchers. If they agreed to let their students take part in the study, they sent information sheets out to parents/guardians of students. Similar information sheets were sent to parents/guardians who had previously indicated that they would be happy to be contacted about future research studies on 
Table 1: Demographic information, questionnaire measures and VAS anxiety ratings. Values in parentheses are standard deviations

\begin{tabular}{|c|c|c|c|c|c|}
\hline & \multicolumn{3}{|c|}{ Training Valence } \\
\hline \multirow{2}{*}{\multicolumn{2}{|c|}{$N$}} & Overall & Neutral & Positive & Positive (24-hr) \\
\hline & & 69 & 23 & 24 & 22 \\
\hline \multicolumn{2}{|c|}{ Female-identified } & $79.7 \%$ & $100 \%$ & $75.0 \%$ & $63.6 \%$ \\
\hline \multicolumn{2}{|c|}{ Mean Age } & $15.68(1.53)$ & $15.48(0.51)$ & $16.70(0.64)$ & $14.82(2.20)$ \\
\hline \multirow[t]{6}{*}{ Ethnicity } & White & $85.5 \%$ & $95.7 \%$ & $70.8 \%$ & $90.9 \%$ \\
\hline & Asian & $7.2 \%$ & $4.3 \%$ & $12.5 \%$ & $4.5 \%$ \\
\hline & Black & $1.4 \%$ & $0 \%$ & $4.2 \%$ & $0 \%$ \\
\hline & Mixed & $4.3 \%$ & $0 \%$ & $8.3 \%$ & $4.5 \%$ \\
\hline & Other & $0 \%$ & $0 \%$ & $0 \%$ & $0 \%$ \\
\hline & Did not report & $1.4 \%$ & $0 \%$ & $4.2 \%$ & $0 \%$ \\
\hline \multicolumn{2}{|c|}{ Anxiety symptoms (STAIC) } & $37.18(7.15)$ & $37.87(6.29)$ & $38.43(6.39)$ & $34.95(8.60)$ \\
\hline \multicolumn{2}{|c|}{ Interpretation bias (ASSIQ) } & $2.1(1.67)$ & $2.65(1.90)$ & $2.04(1.30)$ & $1.73(1.72)$ \\
\hline \multirow{3}{*}{$\begin{array}{l}\text { Anxiety } \\
\text { VAS }\end{array}$} & Pre-training & $1.98(1.67)$ & $2.04(1.40)$ & $2.45(2.08)$ & $1.42(1.34)$ \\
\hline & Post-training & $1.55(1.52)$ & $2.00(1.58)$ & $1.51(1.62)$ & $1.13(1.28)$ \\
\hline & Pre-Test & $1.25(1.40)$ & $1.63(1.57)$ & $1.31(1.54)$ & $0.79(0.89)$ \\
\hline \multirow{3}{*}{$\begin{array}{l}\text { Positive Affect } \\
\text { VAS }\end{array}$} & Pre-training & $6.45(1.36)$ & $6.36(1.19)$ & $6.20(1.32)$ & $6.81(1.56)$ \\
\hline & Post-training & $6.09(1.75)$ & $5.68(1.49)$ & $6.06(1.73)$ & $6.54(1.98)$ \\
\hline & Pre-Test & $6.15(1.81)$ & $5.62(1.64)$ & $6.18(1.94)$ & $6.69(1.78)$ \\
\hline \multirow{3}{*}{$\begin{array}{l}\text { Low Mood } \\
\text { VAS }\end{array}$} & Pre-training & $1.15(1.20)$ & $1.50(1.24)$ & $1.09(1.31)$ & $0.84(0.95)$ \\
\hline & Post-training & $1.38(1.44)$ & $1.87(1.19)$ & $1.28(1.53)$ & $0.97(1.50)$ \\
\hline & Pre-Test & $1.57(1.85)$ & $2.07(1.54)$ & $1.62(2.13)$ & $1.00(1.77)$ \\
\hline
\end{tabular}

behalf of their children. In all cases, the guardians/parents of participants gave fully informed consent and participants provided additional assent to taking part. Prior to providing assent/consent, participants/guardians were made aware that their data would be stored securely and anonymised in both hard and soft copy in the University of Oxford. Participants and guardians were also made aware at this point that their data could be used--in this anonymised format - as part of academic work and research either within the University of Oxford or without, and/or as the basis for journal articles and scientific presentations. Participants and guardians were made aware that they were free to withdraw their consent or data at any point during or following the study.

\section{Results}

\section{Pre-training measures}

Gender, ethnicity and raw means for age, anxiety and interpretation bias scores are given in Table 1. VAS anxiety ratings were found to be positively skewed and non-normally distributed by skewness and Kolmogorov-Smirnov tests. These scores were transformed to better fit normality before inclusion in parametric tests reported below; $\ln (x+1)$ transformations were used to account for the fact that VAS ratings were in some cases zero. All other scores were normally distributed.

Univariate ANOVAs revealed significant group differences in age between the three training conditions (neutral, Same-Session positive, 24-Hour positive): $F(2,65)=11.50, p<.001, \eta^{2}=.261$. Post-hoc comparisons with Bonferroni corrections revealed that participants in the Same-Session positive group were significantly older than in both the neutral $(\mathrm{p}=.009)$ and 24-Hour positive groups $(p<.001)$. Pearson $\chi^{2}$ tests found a significant difference in gender between the three training groups: $\chi^{2}(2)=9.70, p=.008$, with a higher proportions of female participants in the neutral control group relative to the two positive training groups (which were equivalent in gender proportions). No significant differences between groups emerged for proportions of different ethnicities. Crucially, none of the other pre-training measures of anxiety symptom scores or interpretation bias differed 
across groups.

\section{Training Performance}

ANOVAs were conducted to examine performance during training. Separate ANOVAs were conducted for 1) accuracy and 2) reaction times of responses to comprehension questions over training. These contained Training Block $(1,2,3,4,5)$ as a 5-level within-subjects factor and Group (neutral, Same-Session positive, 24-Hour positive) as a 3-level between-subjects factor. Accuracy of responses to comprehension questions did not show significant main effects of either Block or Group, nor any interaction between them. Reaction time decreased significantly over training $\left(F(4,260)=17.17, p<.001, \eta^{2}=.203\right)$, but there was no between-groups difference, nor any Blockby-Group interaction.

\section{Interpretation Bias Test}

A $2 \times 2 \times 3$ ANOVA was first conducted with Probe-Type (target, foil) and Probe-Valence (positive, negative) as 2-level within-subjects factors, and group (neutral, Same-Session positive, 24-Hour positive) as a 3-level between-subjects factor. This yielded a significant 3-way ProbeType-by-Probe-Valence-by-Group interaction: $F(2,66)=4.66, p=.013, \eta^{2}=.121$. To decompose this interaction, two separate $2 \times 2 \times 2$ ANOVAs were conducted, one comparing neutral control training to the Same-Session positive CBM-I paradigm, and one comparing the Same-Session and 24-Hour positive CBM-I groups. These distinctions were made along the lines of our a priori research questions - namely following the interactions between probe type, probe valence and training group in Lothmann et al. (2011) and Lau et al (2011). A third $2 \times 2 \times 2$ ANOVA comparing the neutral control group to the 24-Hour positive training group was then conducted to verify any differences seen between neutral and positive training in the preceding analyses.

\section{Positive CBM-I vs. Neutral Control}

A $2 \times 2 \times 2$ ANOVA was conducted including Probe-Type (target, foil) and Probe-Valence (negative, positive) as 2-level within-subjects factors, and Training-Group (neutral control, SameSession CBM-I) as a 2-level between-subjects factor. This gave a significant 3-way Probe-Type-byProbe-Valence-by-Training-Group interaction: $\mathrm{F}(1,45)=5.12, \mathrm{p}=.029,=.102$.

Targets and Foils were analysed separately to decompose the 3 -way interaction, in two further $2 \times 2$ mixed-model ANOVAs. Targets showed a significant Probe-Valence-by-Training-Group interaction: $F(1,45)=7.08, p=.011, \eta^{2}=.101$, illustrated in Figure 2. Post-hoc $t$-tests showed Negative Targets were endorsed less strongly by the Same-Session positive CBM-I group compared to the neutral group: $p<.029, d=0.67$. This difference remained significant after Tukey corrections for multiple comparisons. No other significant between-group comparisons were found. There was a significant main effect of Probe-Valence: $F(1,45)=18.09, p<.001, \eta^{2}=.258$, but no significant main effect of Training-Group. Paired-sample $t$-tests were used to compare similarity ratings for positive and negative targets individually within each of the two training groups. These showed a significant difference between positive and negative targets in the Same-Session positive group: $t(23)=4.27, p<.001, d=1.78$, such that positive targets were rated as more similar to the original ambiguous scenario than were negative targets. There were no differences between positive and negative target ratings in the neutral group: $t(22)=1.39, p=.179$ (Figure 2). Again comparisons remained significant after Tukey corrections.

Foils also showed a main effect of Probe-Valence: $F(1,45)=22.77, p<.001, \eta^{2}=.327$. Positive foils $(\bar{x}=2.33, s d=0.42)$ were endorsed more strongly than negative foils $(\bar{x}=1.97, s d=0.40)$. In contrast to targets, there was no significant Valence-by-Training-Group interaction, suggesting that there were no differential endorsements of positive and negative foils as a function of CBM-I training.

Three $2 \times 2 \times 2$ ANCOVAs were conducted, each including one of 3 additional measures (age, gender, trait anxiety) as a covariate, again with Probe-Type and Probe-Valence as within-subjects factors and Training-Group as a between-subjects factor. No new main or interaction effects were 


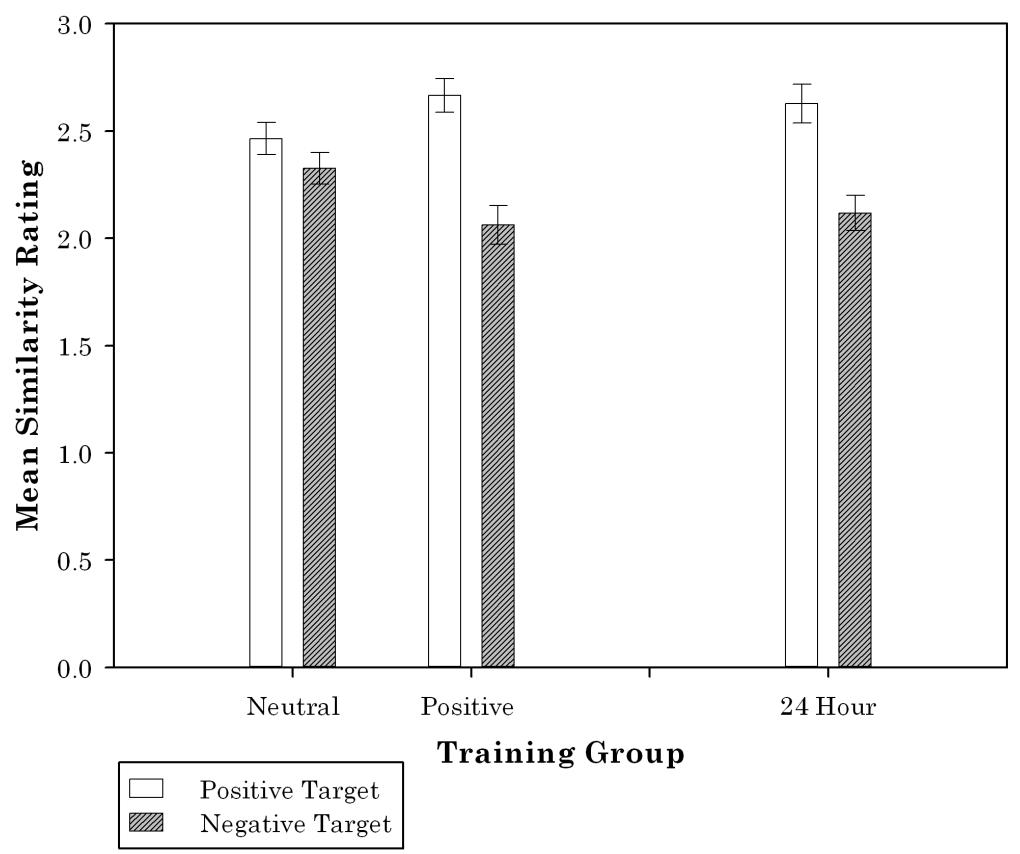

Figure 2: Endorsement of target probes in the Interpretation Bias Test for neutral control training, CBM-I training tested immediately after training (Same-session CBM-I), and CBM-I training tested 24 hours following training (24 Hour CBM-I). Asterisks denote significant differences at the $p<.05$ level.

observed in the analyses including age as a covariate. Including gender gave rise to a significant Probe-Type-by-Gender interaction: $F(1,44)=9.19, p=.004, \eta^{2}=.166$. As above, targets were endorsed more strongly than foils, but comparing Target-Foil difference scores showed this difference was greater in males than females: $t(45)=3.17, p=.003, d=0.944 ; \bar{x}_{\text {female }}=0.18, s d=0.28$; $\bar{x}_{\text {male }}=0.58, s d=0.36$. Owing to significant differences in proportions of genders across the training groups demonstrated by the $\chi^{2}$ tests reported above, analyses were also replicated when including only female participants in the sample. Running analyses while excluding male participants did not change the significance of any findings. When including trait anxiety score as a covariate, a significant Probe-Valence-by-Anxiety interaction emerged: $F(1,43)=6.75, p=.013, \eta^{2}=.100$. Further examination with regression analyses showed that the higher trait anxiety score, the lower similarity ratings were for positively valenced probes: $\beta=-.349, p=.018$. There was no significant relationship between trait anxiety scores and negative probes.

\section{Same Session Positive CBM-I vs. 24 Hour Follow-Up}

A second $2 \times 2 \times 2$ ANOVA included Probe-Type (target, foil) and Probe-Valence (positive, negative) as 2-level within-subjects factors, and Follow-Up (Same-Session, 24-Hour) as a 2-level between-subjects factor. Importantly, there was no significant main effect of Follow-Up $(\mathrm{F}(1,44)$ $=0.02, \mathrm{p}=.886)$, indicating similar pattern of results across the CBM-I Same-Session and 24Hour groups. Similarity ratings were higher for positive than negative statements (main effect of Probe-valence: $F(1,44)=39.42, p<.001, \eta^{2}=.472$ ) but a significant 3 -way Probe-Type-by-ProbeValence-by-Follow-Up interaction also emerged: $F(1,44)=8.10, p=.007, \eta^{2}=.155$. 
To decompose the 3 -way interaction, two separate $2 \times 2$ ANOVAs were conducted for targets and foils respectively. Both targets and foils showed main effects of Probe-Valence as in the $2 \times 2 \times 2$ ANOVA, but again there were no significant main effects of Follow-Up, nor any significant ProbeValence-by-Follow-Up interactions. Given the lack of any 2-way interactions, post-hoc $t$-tests were conducted to examine any possible differences between Follow-Up-Groups in light of the 3-way interaction reported above. Same-Session and 24-Hour groups did not differ significantly on similarity ratings for positive targets $(t(44)=.33, p=.744)$ nor negative targets $(t(44)=-0.45, p=.653)$, nor on difference scores between positive and negative targets (i.e. Positive Target Ratings-Negative Target Ratings): $t(44)=0.49, p=.630$-see Figure 2 .

As above, the $2 \times 2 \times 2$ ANOVA was replicated in a series ANCOVAs, including age, gender, and trait anxiety as covariates in separate analyses. No new main or interaction effects were observed when including age as covariate. The only change to results upon including gender was the emergence of a Probe-Type-by-Gender interaction: $F(1,43)=6.27, p=.016, \eta^{2}=.127$. Again this effect was such that the extent to which targets were more strongly endorsed than foils was higher in males $(\bar{x} M=0.42, s d=0.32)$ than females $(\bar{x}=0.19, s d=0.26): t(44)=2.50, p=.016, d=0.754$. Again, all analyses were also repeated when including only female participants in the sample, but this did not change the significance of any findings. The only change to results upon including trait anxiety as a covariate was a novel Valence-by-Anxiety interaction: $F(1,40)=12.94, p=.001, \eta^{2}=.168$. Regression analyses showed that, as above, weaker endorsements of positive probes were associated with higher scores on the STAIC $(\beta=-.452, p=.002)$. Higher trait anxiety scores were additionally associated with stronger endorsement of negative probes: $\beta=.304, p=.047$.

\section{Neutral Control vs. 24-Hour Positive Training}

The previous two analyses demonstrated significant effect of training on interpretations, and an absence of significant decreases in post-training interpretations, respectively. A third set of comparisons were conducted to examine if interpretations were significantly more positive 24 hours following training, compared to the neutral control. A $2 \times 2 \times 2$ ANOVA was conducted including Probe-Type (target, foil) and Probe-Valence (negative, positive) as 2-level within-subjects factors, and Training-Group (neutral control, 24-Hour positive) as a 2-level between-subjects factor. This gave a non-significant 3-way Probe-Type-by-Probe-Valence-by-Training-Group interaction: $F(1,43)=.23, p=.631$.

Targets and Foils were again analysed separately in two further $2 \times 2$ mixed-model ANOVAs, following the protocol of earlier studies (Lau et al., 2011; Lothmann et al., 2011). Targets showed a significant Probe-Valence-by-Training-Group interaction: $F(1,43)=4.96, p=.031, \eta^{2}=.079$. There was a significant main effect of Probe-Valence: $F(1,43)=27.88, p<.001, \eta^{2}=.358$, but no significant main effect of Training-Group. Paired-sample $t$-tests were used to compare similarity ratings for positive and negative targets individually within each of the two training groups. These showed a significant difference between positive and negative targets in the 24-Hour positive group: $t(21)=3.80, p=.001, d=1.66$, such that positive targets were rated as more similar to the original ambiguous scenario than were negative targets. There were no differences between positive and negative target ratings in the neutral group: $t(22)=1.39, p=.179, d=.290$. Comparisons remained significant after Tukey corrections.

Foils also showed a main effect of Probe-Valence: $F(1,43)=38.07, p<.001$, partial $^{2}=.470$. Positive foils $(\bar{x}=2.33, s d=0.42)$ were endorsed more strongly than negative foils $(\bar{x}=1.97, s d=$ 0.40). There was a significant Valence-by-Training-Group interaction: $F(1,43)=7.59, p=.009, \eta^{2}=$ .086, suggesting differential endorsements of positive and negative foils as a function of CBM-I training. Positive foils were more strongly endorsed than negative foils in both training condition, but positive foils were more strongly endorsed in the positive training group compared to the neutral control, and vice versa for negative foils. However the $\eta^{2}$ score for this interaction shows that this was a small effect.

ANCOVAs were again run including age, gender and STAIC score in three separate analyses. No new main or interaction effects were observed when including age or gender in the analyses. As in previous analyses, a 2 -way valence-by-STAIC interaction emerged $F(1,40)=10.74, p=.002, \eta^{2}=$ 


\section{VAS Ratings}

To investigate the effects of training condition on changes in state anxiety ratings from before to after training, a $2 \times 3$ ANOVA was conducted with Time (pre-training, post-training) as a 2-level within-subjects factor, and Group (neutral control, Same-Session positive, 24-Hour positive) as a 3level between-subjects factor. A main effect of time was found $\left(F(1,65)=7.78, p=.007, \eta^{2}=.101\right)$ such that state anxiety ratings decreased from pre-training $(\bar{x}=1.98, s d=1.67)$ to post-training $(\bar{x}=1.55, s d=1.52)$, though this effect was small. There was no main effect of Group, nor any significant Time-by-Group interactions. Post-hoc comparisons showed no significant differences between VAS1 or VAS2 scores between any two groups in the analyses.

A univariate ANOVA with Group (neutral control, Same-Session Positive, 24-Hour Positive) as a 3-level factor was used to check for between-groups differences in anxiety at pre-test. This was done to ensure that any between-groups differences observed on the Interpretation Bias Test were not ascribable to confounding mood-congruence effects rather than the result of modified interpretations following training. No significant differences were found between groups, and post-hoc comparisons showed no differences between any 2 groups in the analyses.

The ANOVAs were re-run including STAIC scores, gender and age in separate covariate analyses. The inclusion of these factors did not affect the significance of any results, nor did it give rise to any significant novel interaction effects. ANOVAs were repeated for positive affect and low mood VAS scores. Neither of these sets of analyses showed any significant between-groups differences, nor any differential change as a result of training valence.

\section{Discussion}

The current study aimed to examine whether the effects of positive CBM-I training on the interpretation of novel ambiguous scenarios persisted for 24 hours in an adolescent sample. Results replicated previous findings that CBM-I training is acquired effectively in adolescents (Lau et al., 2011; Lothmann et al., 2011; Salemink \& Wiers, 2011). Results from the Interpretation Bias Test suggest that biases congruent with positive training material were applied to novel ambiguous social scenarios, relative to neutral control training. Post-hoc comparisons replicated previous adolescent findings that negative targets were endorsed less strongly as interpretations of novel ambiguous situations following positive training (Fu et al., 2012; Lau et al., 2011; Lester et al., 2011a, 2011b; Lothmann et al., 2011; Salemink \& Wiers, 2011). This contributes to a body of work suggesting that positive CBM-I training may be effective in suppressing maladaptive negative patterns of social cognitive bias. Findings of no difference between an Interpretation Bias Test administered during the same session as training, and one administered 24 hours later suggest that positive training effects from the CBM-I paradigm in adolescents are comparable when measured either immediately following training or a day later. This replicates previous adult findings (Mackintosh et al., 2006; Yiend et al., 2005). Persistence over 24 hours marks a useful milestone for a paradigm that would be intended in its final form to contribute to long-lasting changes in cognition and behaviourbuffering against risk factors for anxiety over development (Hoppitt et al., 2010; MacLeod \& Holmes, 2012) - and supports other such encouraging early research into the persistence of CBM-I training effects in youth (Vassilopoulos et al., 2009, 2012). The results for target probes (which are most indicative of interpretive biases: Mathews \& Mackintosh, 2000) did not differ between the two sets of comparisons - that is, between the "Neutral vs. Same-Session Positive" and the "Neutral vs. 24-Hour Positive" analyses. This finding supports the assertion that training effects for interpretive biases as a result of CBM-I persist over a 24 hour period in adolescents.

Self-reported anxiety ratings decreased across the whole sample over the course of CBM-I training. However, no differential effects on training were seen on state measures of state anxiety, positive affect or low mood. These findings replicate previous studies to have shown no evidence of mood change as a direct effect of training in adolescents (Fu et al., 2012; Lester et al., 2011b; Salemink \& 
Wiers, 2011; Vassilopoulos, Moberly, \& Zisimatou, 2013). In doing so they also contrast with adolescent findings of immediate mood changes seen in various studies (Lester et al., 2011a; Lothmann et al., 2011; Vassilopoulos et al., 2009, 2012). Other adolescent work has suggested that such mood effects might only be observed in individuals with pre-existing high anxiety or low self-efficacy (Lau et al., 2011; Lothmann et al., 2011), or after multiple instances of training (Vassilopoulos et al., 2009, 2012). Placed in a wider context with adult findings, it is possible that post-training differences in anxiety would only be constructed through interactions with stressors and other features of the environment (Hoppitt et al., 2010; Mathews, 2012). Previous work has shown that changes in state anxiety as a result of CBM-I training are most readily observable in terms of differential responses to experimental or environmental stressors, rather than simply testing mood directly following training (Hallion \& Ruscio, 2011). More recent work has suggested that this is equally true of adolescent as of adult samples (Lau, Belli, et al., 2013). Examining post-training anxiety effects with regards to reactivity to environmental stressors or other processes (e.g. appraisal of intervening life events, as in Telman, Holmes, \& Lau, 2013) would be an interesting development if differential post-training anxiety changes could be demonstrated under such circumstances.

Current findings also bear upon the methodology of future research. Typically past CBM-I research, including adolescent work, has compared positive to negatively valenced CBM-I training in typically-developing samples, so as to maximise the observed effects (Lothmann et al., 2011; Mathews \& Mackintosh, 2000). However the current data show that differences are detectable between positive CBM-I and neutral control training. While this finding might have limited bearing upon future experimental studies, future work that aims to clarify any clinical utility of CBM-I training requires the development of suitable control conditions. Such work should avoid using negative bias training in clinical or symptomatic samples for ethical reasons; such individuals may selectively fixate upon negative information within the paradigm (Mogg \& Bradley, 1998; Williams, 2004). To prevent against unduly exposing clinical or at-risk populations to material that may compound their negative interpretative biases, it seems desirable to develop a viable control for positive CBM-I that is not predominantly comprised of negative training material. Based on the results from the present study, the neutral training group could be an acceptable control.

The ANCOVAs that included trait anxiety as a covariate showed that higher trait anxiety was associated with weaker endorsement of positive probes, but that this association did not interact with training. This association replicates findings from previous work of links between interpretation biases and trait emotional symptoms (Amir et al., 1998; Hadwin et al., 1997; Mathews \& Mackintosh, 2000; Muris, Huijding, Mayer, \& Hameetman, 2008; Salemink \& Wiers, 2011). These findings show continuity with negative and non-positive interpretive biases seen in clinical adult populations (Blackwell \& Holmes, 2010; Eysenck et al., 1991; Mathews, Richards, \& Eysenck, 1989). That is to say that similar associations between the valence of interpretations and trait anxiety scores are seen regardless of whether an individual has clinically significant internalising or emotional disorders. The current study also provides further evidence that strength of interpretive bias is associated with strength of symptoms in adolescence as well as in adulthood (Hadwin \& Field, 2010). These data suggest that to the extent that CBM-I is effective, it is no less (or more) effective in symptomatic individuals.

It is worth mentioning that although the pattern of results was very similar for the comparisons of the neutral control group to each of the same-session and 24-Hour positive training groups, there were minor differences. Namely, the latter comparison showed a significant Probe-Valence-by-TrainingGroup interaction for foils. This was in the expected direction (i.e. positively trained individuals endorsed negative targets less strongly than did the control training group), but this interaction was not significant in the comparison of neutral control training to the positive same-session group. The significant interaction for foils also explains the fact that the 3-way Probe-Valence-by-ProbeType-by-Training-Group was non-significant in the latter set of analyses. In the neutral-control vs. positive same-session comparisons this 3-way interaction was, by contrast, significant - the key difference being that the Probe-Valence-by-Training-Group interaction was not significant in the neutral vs. positive same session comparisons. It is also worth noting that there were two stages of removal between the neutral control and 24-hour positive groups, i.e. the two groups underwent different training valences, and received post-training interpretation tests in different circumstances. 
Thus, the first two sets of analesys (positive vs. negative in same session; same session vs. 24 hour following positive training) were more theoretically watertight. That is, each only changed one variable between comparison groups, allowing for keener inferences to be made.

\section{Limitations}

Though not necessarily a limitation, the 3-arm trial used in the current study was one alternative among a variety of approaches that could have been taken. For example an additional 24-hour neutral control group would allow for symmetrical comparisons of variations in interpretive bias over a 24-hour period in the absence of positive training, which is not possible with the current design. Such a comparison would be of theoretical merit as it would control for any external influences on interpretive bias - for instance intervening daily experiences, or natural variations in mood or cognitive ability. Indeed, interaction with daily life events has been identified as a crucial consideration for the proposed mechanism of action for any application of CBM-I training (Hoppitt et al., 2010). Including a 24-hour control group would rule out such potential confounding effects. An alternative would have been to use a repeated measures design, in order to track variation in interpretive biases over time. However, in order to perform such an experiment in future there would be a need to develop a new index of interpretive bias, as the Interpretive Bias Test becomes extremely transparent in aim if administered more than once.

The pre-training measure of interpretation used in the current study showed relatively poor internal reliability $(\alpha=.56)$. This does not bear too heavily on the current results, as the interpretation questionnaire was only used to test for pre-training between-groups differences, finding null results on either this measure or anxiety scores (which are highly correlated with negative interpretive biases). However, a low reliability score suggests that the items on the questionnaire do not provide a coherent index of 'interpretation bias'. Since, for example, certain items may be more or less indicative of interpretation bias in an unreliable questionnaire, scores from such a measure may mask actual levels of interpretation bias. In such a case, the questionnaire would be inappropriate for testing differences in interpretation bias between groups. Additionally the low reliability would be an issue for future work that would seek to measure interpretation biases, and especially any studies attempting to measure changes in such a measure. Given that administering the Interpretation Bias Test both before and after training might confound results by making the purpose of the test transparent, more efforts are needed in future to develop accurate and reliable alternative measures of interpretation bias. Previous studies have in fact shown discrepancies between the Interpretation Bias Test and other indices of interpretation bias currently in use (Fu et al., 2012; Salemink, van den Hout, \& Kindt, 2009). A specific challenge for future research is therefore to develop a measure of interpretive bias that shows good construct validity in predicting/correlating with the Interpretation Bias Test, without comprising the element of surprise that is necessary for the test's validity.

The study is limited in its generalisation beyond the non-clinical sample of the current experiment. Anxious symptom scores in the current sample were similar to normative adolescents' scores on these measures (Spielberger, 1973), as would be expected from a sample drawn from a typicallydeveloping population. As such the present study is limited in its generalisability to clinical or sub-clinical high-symptomatic groups, and so findings remain limited in their applicability to meaningful implementation of CBM-I protocols as potential interventions against the development of emotional disorders in adolescence.

The current sample was disproportionately female-heavy, and both age and gender make-up differed between groups. Though neither of these aspects of the sample is desirable, they were unfortunately the result of random (rather than quota/stratified) sampling measures used for group assignment. It is encouraging that no differences to the ANOVAs reported in the section above were found upon including either gender or age as covariates in the analysis, nor when solely analysing female participants. Future research should seek to negate the need for such post-hoc checks and analyses by controlling for these factors at the outset of the experiment. 


\section{Conclusion}

In summary, the current study showed that the effects of positive CBM-I training upon interpretation (compared to control) persist over a 24 hour period. The current study did not find any commensurate changes in anxiety as a result of these interpretation effects, but findings identify a path for future bias training research examining the role of stress reactivity in such scenarios. 


\section{Compliance with Ethical Standards}

Disclosure of Potential Conflicts-of-Interest SRB and JY-FL declare that they have no conflict of interests. This study was funded through an Economic and Social Research Council 1+3 studentship awarded to SRB. Neither SRB nor JY-FL has any other financial disclosure to make. The data have not been previously published or presented at any conference or academic meeting, though they have appeared (in a different form) in SRBs doctoral thesis.

\section{Informed Consent}

All procedures followed were in accordance with the ethical standards of the responsible committee on human experimentation (institutional and national) and with the Helsinki Declaration of 1975 , as revised in 2000 (5). Informed consent was obtained from all patients for being included in the study 


\section{References}

American Psychiatric Association. (2000). Diagnostic and Statistical Manual of Mental Disorders (text revision) (4th ed.). Washington DC: American Psychiatric Publishing Inc.

Amir, N., Foa, E. B., \& Coles, M. E. (1998). Negative interpretation bias in social phobia. Behaviour research and therapy, 36, 945-957.

Blackwell, S. E., \& Holmes, E. A. (2010). Modifying interpretation and imagination in clinical depression: A single case series using cognitive bias modification. Applied Cognitive Psychology, 24, 338-350. doi:10.1002/acp.1680

Blakemore, S.-J. (2008). The social brain in adolescence. Nature reviews. Neuroscience, 9(4), 267-77. doi:10.1038/nrn2353

Blakemore, S.-J., \& Choudhury, S. (2006). Development of the adolescent brain: implications for executive function and social cognition. Journal of Child Psychology and Psychiatry, 47(3), 296-312. doi:10.1111/j.1469-7610.2006.01611.x

Cohen Kadosh, K., Linden, D. E. J., \& Lau, J. Y. F. (2013). Plasticity during childhood and adolescence: innovative approaches to investigating neurocognitive development. Developmental Science, 16(4), 574-583. doi:10.1111/desc.12054

Costello, E. J., Egger, H. L., \& Angold, A. (2005). The Developmental Epidemiology of Anxiety Disorders: Phenomenology, Prevalence and Comorbidity. Child and Adolescesnt Psychiatric Clinics of North America, 14, 631-648.

Creswell, C., OConnor, T. G., \& Brewin, C. (2006). A Longitudinal Investigation of Maternal and Child Anxious Cognitions. Cognitive Therapy and Research, 30(2), 135-147. doi:10.1007/s10608006-9021-1

Creswell, C., Schniering, C. A., \& Rapee, R. M. (2005). Threat interpretation in anxious children and their mothers: comparison with nonclinical children and the effects of treatment. Behaviour research and therapy, 43(10), 1375-1381.

Eysenck, M. W., Mogg, K., May, J., Richards, A., \& Mathews, A. (1991). Biased interpretation of ambiguous sentences related to threat in anxiety. Journal of Abnormal Psychology, 100, 144-150.

Field, A. P., \& Lester, K. J. (2010). Learning of Information Processing Biases in Anxious Children and Adolescents. In J. A. Hadwin \& A. P. Field (Eds.), Information Processing Biases and Anxiety: A Developmental Perspective (pp. 253278). Chichester, West Sussex/Oxford/Malden MA: Wiley-Blackwell.

Fu, X., Du, Y., Au, S., \& Lau, J. Y. F. (2013). Reducing Negative Interpretations in Adolescents with Anxiety Disorders: A Preliminary Study Investigating the Effects of a Single Session of Cognitive Bias Modification Training. Developmental Cognitive Neuroscience, 4, 29-37. doi:10.1016/j.den.2012.11.003

Gregory, A. M., Caspi, A., Moffitt, T. E., Koenen, K., Eley, T. C., \& Poulton, R. (2007). Juvenile mental health histories of adults with anxiety disorders. American Journal of Psychiatry, 164(2), 301-308. doi:164/2/301 [pii] 10.1176/appi.ajp.164.2.301

Hadwin, J. A., \& Field, A. P. (2010). An Introduction to the Study of Information Processing Biases in Childhood Anxiety: Theoretical and Methodological Issues. In J. A. Hadwin \& A. P. Field (Eds.), Information Processing Biases and Anxiety: A Developmental Perspective (pp. 117). Chichester, West Sussex/Oxford/Malden MA: Wiley-Blackwell.

Hadwin, J. A., Frost, S., French, C. C., \& Richards, A. (1997). Cognitive processing and trait anxiety in typically developing children: evidence for an interpretation bias. Journal of Abnormal Psychology, 106, 486-490.

Haller, S. P. W., Cohen Kadosh, K., \& Lau, J. Y. F. (2013). A developmental angle to understanding the mechanisms of biased cognitions in social anxiety. Frontiers in Human Neuroscience, 7, 846 .

Hallion, L. S., \& Ruscio, A. M. (2011). A meta-analysis of the effect of cognitive bias modification on anxiety and depression. Psychological Bulletin, 137(6), 940-58. doi:10.1037/a0024355

Hettema, J. M., Prescott, C. A., \& Kendler, K. S. (2003). The effects of anxiety, substance use and conduct disorders on risk of major depressive disorder. Psychological Medicine, 33(8), 14231432. doi:10.1017/S0033291703008365 
Holmes, E. A., Lang, T. J., \& Shah, D. M. (2009). Developing Interpretation Bias Modification as a "Cognitive Vaccine" for Depressed Mood: Imagining Positive Events Makes You Feel Better Than Thinking About Them Verbally. Journal of Abnormal Psychology, 118(1), 76-88. doi: $10.1037 / \mathrm{a} 0012590$

Holmes, E. A., Mathews, A., Dalgleish, T., \& Mackintosh, B. (2006). Positive Interpretation Training: Effects of Mental Imagery Versus Verbal Training on Positive Mood. Behavior Therapy, 37(3), 237-247. doi:10.1016/j.beth.2006.02.002

Hoppitt, L., Mathews, A., Yiend, J., \& Mackintosh, B. (2010). Cognitive mechanisms underlying the emotional effects of bias modification. Applied Cognitive Psychology, 24(3), 312-325. doi:10.1002/acp.1678

Kim-Cohen, J., Caspi, A., Moffitt, T. E., Harrington, H., Milne, B. J., \& Poulton, R. (2003). Prior juvenile diagnoses in adults with mental disorder-Developmental follow-back of a prospectivelongitudinal cohort. Archives of General Psychiatry, 60, 709-717.

Lang, T. J., Blackwell, S. E., Harmer, C. J., Davison, P., \& Holmes, E. A. (2012). Cognitive Bias Modification Using Mental Imagery for Depression: Developing a Novel Computerized Intervention to Change Negative Thinking Styles. European Journal of Personality, 157, 145-157. doi:10.1002/per.855

Lau, J. Y. F. (2013). Cognitive bias modification of interpretations: A viable treatment for child and adolescent anxiety? Behaviour Research and Therapy, 51(10), 614-622.

doi:10.1016/j.brat.2013.07.001

Lau, J. Y. F., Belli, S. R., \& Chopra, R. B. (2013). Cognitive bias modification training in adolescents reduces anxiety to a psychological challenge. Clinical Child Psychology and Psychiatry, 18(3), 322-333. doi:10.1177/1359104512455183

Lau, J. Y. F., Molyneaux, E., Telman, M., \& Belli, S. (2011). The Plasticity of Adolescent Cognitions: Data from a Novel Cognitive Bias Modification Training Task. Child Psychiatry \& Human Development, 42(6), 679-693. doi:10.1007/s10578-011-0244-3

Lau, J. Y. F., Pettit, E., \& Creswell, C. (2013). Reducing children's social anxiety symptoms: Exploring a novel parent-administered cognitive bias modification training intervention. Behaviour Research and Therapy, 51(7), 333-337. doi:http://dx.doi.org/10.1016/j.brat.2013.03.008

Laurent, J., Catanzaro, S. J., Joiner, T. E., Rudolph, K. D., Potter, K. I., Lambert, S., ... Gathright, T. (1999). A measure of positive and negative affect for children: scale development and preliminary validation. Psychological Assessment, 11, 326-338.

Lester, K. J., Field, A. P., \& Muris, P. (2011a). Experimental modification of interpretation bias about animal fear in young children: effects on cognition, avoidance behavior, anxiety vulnerability, and physiological responding. Journal of Clinical Child and Adolescent Psychology, 40(6), 864-877. doi:10.1080/15374416.2011.618449

Lester, K. J., Field, A. P., \& Muris, P. (2011b). Experimental modification of interpretation bias regarding social and animal fear in children. Journal of Anxiety Disorders, 25(5), 697-705. doi:10.1016/j.janxdis.2011.03.006

Lothmann, C., Holmes, E. A., Chan, S. W. Y., \& Lau, J. Y. F. (2011). Cognitive bias modification training in adolescents: effects on interpretation biases and mood. Journal of Child Psychology and Psychiatry, 52(1), 24-32. doi:10.1111/j.1469-7610.2010.02286.x

Mackintosh, B., Mathews, A., Yiend, J., Ridgeway, V., \& Cook, E. (2006). Induced biases in emotional interpretation influence stress vulnerability and endure despite changes in context. Behavior Therapy, 37(3), 209-222. doi:10.1016/j.beth.2006.03.001

MacLeod, C. M., \& Holmes, E. A. (2012). Cognitive Bias Modification: An Intervention Approach Worth Attending To. American Journal of Psychiatry, 169(2), 118-120. doi:10.1176/appi.ajp.2011.11111682

Mathews, A. (2012). Effects of modifying the interpretation of emotional ambiguity. Journal of Cognitive Psychology, 24(1), 92-105.

Mathews, A., \& Mackintosh, B. (2000). Induced emotional interpretation bias and anxiety. Journal of Abnormal Psychology, 109(4), 602-615. doi:10.1037/0021-843x.109.4.602

Mathews, A., \& MacLeod, C. (2002). Induced processing biases have causal effects on anxiety. Cognition \& Emotion, 16(3), 331-354. 
Mathews, A., Richards, A., \& Eysenck, M. W. (1989). Interpretation of homophones related to threat in anxiety states. Journal of Abnormal Psychology, 109, 602-615.

Meltzer, H., Gatward, R., Goodman, R., \& Ford, T. (2000). Mental health of children and adolescents in Great Britain. International review of psychiatry, 15(1-2), 185-187. doi:10.1080/0954026021000046155

Moffitt, T. E., Harrington, H., Caspi, A., Kim-Cohen, J., Goldberg, D., Gregory, A. M., \& Poulton, R. (2007). Depression and Generalized Anxiety Disorder. Archives of General Psychiatry, $64,651-660$.

Mogg, K., \& Bradley, B. P. (1998). A cognitive-motivational analysis of anxiety. Behaviour Research and Therapy, 36, 809-848.

Muris, P., Huijding, J., Mayer, B., \& Hameetman, M. (2008). A space odyssey: experimental manipulation of threat perception and anxiety-related interpretation bias in children. Child Psychiatry and Human Development, 39, 469-480. doi:10.1007/s10578-008-0103-Z

Muris, P., Huijding, J., Mayer, B., Remmerswaal, D., \& Vreden, S. (2009). Ground control to Major Tom: experimental manipulation of anxiety-related interpretation bias by means of the "space odyssey" paradigm and effects on avoidance tendencies in children. Journal of Anxiety Disorders, 23(3), 333-340. doi:10.1016/j.janxdis.2009.01.004

Rapee, R. M., Schniering, C. A., \& Hudson, J. L. (2009). Anxiety disorders during childhood and adolescence: origins and treatment. Annual Review of Clinical Psychology, 5, 311-341. doi:10.1146/annurev.clinpsy.032408.153628

Rohde, P., Lewinsohn, P. M., \& Seeley, J. R. (1991). Comorbidity of unipolar depression: II. Comorbidity with other mental disorders in adolescents and adults. Journal of Abnormal Psychology, 100(2), 214-222.

Salemink, E., van den Hout, M., \& Kindt, M. (2009). Effects of positive interpretive bias modification in highly anxious individuals. Journal of Anxiety Disorders, 23, 676683.

Salemink, E., \& Wiers, R. (2011). Modifying Threat-related Interpretive Bias in Adolescents. Journal of Abnormal Child Psychology, 39(7), 967-976. doi:10.1007/s10802-011-9523-5

Spielberger, C. D. (1973). Manual for the state-trait anxiety inventory for children. Palo Alto, CA: Consulting Psychologists Press.

Steinberg, L., \& Monahan, K. C. (2007). Age Differences in Resistance to Peer Influence. Developmental Psychology, 43(6), 1531-1543. doi:10.1037/0012-1649.43.6.1531.Age

Stopa, L., \& Clark, D. M. (2000). Social phobia and interpretation of social events. Behaviour Research and Therapy, 38, 273-283.

Suway, J. G., \& Fox, N. A. (2012). Cognitive Bias Modification: The Effects of Training Paradigms. In J. A. Burack, J. T. Enns, \& N. A. Fox (Eds.), Cognitive Science, Development, and Psychopathology: Typical and Atypical Developmental Trajectories of Attention (pp. 47-69). New York, NY; Oxford: Oxford University Press.

Vassilopoulos, S. P., \& Banerjee, R. (2008). Interpretations and judgments regarding positive and negative social scenarios in childhood social anxiety. Behaviour Research and Therapy, 46(7), 870-876. doi:10.1016/j.brat.2008.03.008

Vassilopoulos, S. P., Banerjee, R., \& Prantzalou, C. (2009). Experimental modification of interpretation bias in socially anxious children: Changes in interpretation, anticipated interpersonal anxiety, and social anxiety symptoms. Behaviour Research and Therapy, 47(12), 1085-1089.

Vassilopoulos, S. P., Blackwell, S. E., Moberly, N. J., \& Karahaliou, E. (2012). Comparing imagery and verbal instructions for the experimental modification of interpretation and judgmental bias in children. Journal of behavior therapy and experimental psychiatry, 43(1), 594601. doi:10.1016/j.jbtep.2011.08.004

Vassilopoulos, S. P., Moberly, N. J., \& Zisimatou, G. (2013). Experimentally modifying interpretations for positive and negative social scenarios in children: a preliminary investigation. Behavioural and Cognitive Psychotherapy, 41(1), 103-116. doi:10.1017/S1352465812000537

Warren, S. L., Emde, R. N., \& Sroufe, L. A. (2000). Internal Representations: Predicting Anxiety From Childrens Play Narratives. Journal of the American Academy of Child E Adolescent Psychiatry, 39(1), 100-107. doi:10.1097/00004583-200001000-00022 
Williams, J. M. G. (2004). Experimental cognitive psychology and clinical practice: autobiographical memory as a paradigm case. In J. Yiend (Ed.), Cognition, Emotion and Psychopathology. Cambridge: Cambridge University Press.

Yiend, J., Mackintosh, B., \& Mathews, A. (2005). Enduring consequences of experimentally induced biases in interpretation. Behaviour Research and Therapy, 43, 779-797. 